\title{
Dose Response Association between Physical Activity and Biological, Demographic, and Perceptions of Health Variables
}

\author{
Paul D. Loprinzi ${ }^{a}$ Hyo Lee ${ }^{b}$ Bradley J. Cardinal ${ }^{b}$ \\ a Department of Exercise Science, Bellarmine University, Louisville, KY, ${ }^{b}$ Department of Sport \\ and Health Sciences, Sangmyung University, Seoul, Korea, ${ }^{C}$ Program in Exercise and Sport \\ Science, School of Biological and Population Health Sciences, College of Public Health and \\ Human Sciences, Oregon State University, Corvallis, OR, USA
}

\author{
Key Words \\ Epidemiology $\cdot$ Exercise $\cdot$ Health benefits
}

\begin{abstract}
Background: Few population-based studies have examined the association between physical activity (PA) and cardiovascular disease risk factors, demographic variables, and perceptions of health status, and we do not have a clear understanding of the dose-response relationship among these variables. Methods: Data from the 2003-2006 National Health and Nutrition Examination Survey was used to examine the dose-response relationship between objectively measured PA and metabolic syndrome (and its individual cardiovascular disease risk factors), demographic variables, and perceptions of health. After exclusions, 5,538 participants 18 years or older were included in the present study, with 2,538 participants providing fasting glucose and 2,527 providing fasting triglyceride data. PA was categorized into deciles. Results: Overall, the health benefits showed a general pattern of increase with each increasing levels of PA. Of the ten PA classifications examined, participants in the highest moderate-to-vigorous physical activity (MVPA) category (at least $71 \mathrm{~min} /$ day) had the lowest odds of developing metabolic syndrome. Conclusion: At a minimum, sedentary adults should strive to meet current PA guidelines (i.e., $150 \mathrm{~min} /$ week of MVPA), with additional positive benefits associated with engaging in three times this level of PA.

(c) 2013 S. Karger GmbH, Freiburg
\end{abstract}


Loprinzi et al.: Dose Response Association Between Physical Activity and Biological, Demographic, and Perceptions of Health Variables

\section{Introduction}

Accounting for over 600,000 deaths in 2006, cardiovascular disease is one of the leading causes of mortality [1]. Individuals with metabolic syndrome are at an increased risk for developing cardiovascular disease [2]. As demonstrated in previous studies, individuals who participate in greater amounts of physical activity are less likely to develop metabolic syndrome and its individual cardiovascular disease risk factors [3-5]. However, few epidemiological studies have examined the association between objectively measured physical activity, metabolic syndrome, and cardiovascular disease risk factors [6, 7].

An exception to this is the study by Metzger et al. [7]. They used data from the 2003-2004 National Health and Nutrition Examination Survey (NHANES) cycle and employed latent class analysis to examine patterns of physical activity associated with metabolic syndrome and cardiovascular risk factors. In comparison to the least active group they identified that those in the four most active groups had the lowest associated odds of experiencing metabolic syndrome and its individual cardiovascular disease risks.

This present study extends upon the work of Metzger et al. [7] by examining the dose response relationship between objectively measured moderate-to-vigorous physical activity (MVPA), metabolic syndrome, and cardiovascular disease risk factors using the two NHANES cycles where objectively measured physical activity was collected (i.e., 2003-2004 and 20052006). Use of both NHANES cycles increased the sample size by as much as 2,080 participants for the non-fasting variables. Moreover, in this study we were specifically interested in assessing the strength of association with metabolic syndrome and cardiovascular disease risk factors using ten incremental physical activity categories for both light-intensity physical activity and MVPA. Gaining an understanding of which specific levels of physical activity are associated with lower risks of metabolic syndrome and its individual cardiovascular disease risk factors may facilitate the development and implementation of physical activity messages and intervention strategies.

Extending the work of Hawkins et al. [8], a secondary objective was to examine levels of objectively measured physical activity by age, gender, and race-ethnicity using all available objectively measured physical activity data. Lastly, a third objective was to examine the doseresponse relationship between objectively measured physical activity and perceptions of physical and mental health status. Although previous studies have reported a link between physical activity and perceived health function, it is not clear as to what level of objectively measured physical activity is most strongly associated with self-perceptions of health. Understanding such information may be useful in the development and implementation of effective physical activity interventions aimed at enhancing health perception as well as in improving a variety of physiological parameters given the association between health perception and indices of cardiovascular disease [9-11].

\section{Material and Methods}

Design and Participants

The NHANES data are collected annually. The data presented herein are from the NHANES 2003-2004 and 2005-2006 cycles. NHANES 2003-2006 used a representative sample of non-institutionalized US civilians selected by a complex, multistage probability design across numerous US geographic locations. The study was approved by the National Center for Health Statistics ethics review board, with informed consent obtained from all participants prior to data collection.

The population for the 2003-2006 NHANES cycles included 20,470 participants. For the present study, we limited our analyses to adults $\geq 18$ years of age, who are not pregnant, had sufficient accelerometry data (i.e. wore accelerometer for at least 4 days and $10 \mathrm{~h} /$ day) and did not have missing data on the covariates 
Loprinzi et al.: Dose Response Association Between Physical Activity and Biological, Demographic, and Perceptions of Health Variables

used in the models (i.e., age, BMI, gender, history of cardiovascular disease or stroke, race-ethnicity, smoking, and socioeconomic status (SES)). After these exclusions, 5,538 participants remained. Among the participants attending the mobile examination center (MEC), approximately half of them were examined in a morning fasting session where fasting biological specimens were obtained. From the morning fasting session, and for the present study, triglycerides and glucose concentrations were analyzed. Data was obtained from 2,538 participants providing fasting glucose and 2,527 providing fasting triglyceride data. 2,405 participants provided complete data for the metabolic syndrome analysis. Participants ranged in age from 18-85 years.

Primary Outcome: Metabolic Syndrome

Consistent with the American Heart Association/National Heart, Lung, and Blood Institute guidelines [12], participants were classified as having metabolic syndrome if they had three or more of the following: i) high waist circumference ( $\geq 102 \mathrm{~cm}$ for men and $\geq 88 \mathrm{~cm}$ for women); ii) high levels of triglycerides ( $\geq 150$ $\mathrm{mg} / \mathrm{dl}$ ); iii) low level of high-density lipoprotein (HDL) cholesterol ( $<40 \mathrm{mg} / \mathrm{dl}$ for men and $<50 \mathrm{mg} / \mathrm{dl}$ for women or self-reporting taking cholesterol-lowering medication); iv) elevated blood pressure ( $\geq 130 \mathrm{~mm}$ Hg systolic or $\geq 85 \mathrm{~mm} \mathrm{Hg}$ diastolic or self-reporting taking hypertensive-lowering medication); and v) elevated fasting glucose ( $\geq 100 \mathrm{mg} / \mathrm{dl}$ or self-reporting taking insulin or pills for diabetes).

\section{Additional Cardiovascular Disease Risk Factors}

At the MEC, blood samples were obtained from the participants. Fasting blood samples were obtained for triglycerides and glucose. Fasting low-density lipoprotein (LDL) cholesterol was not used in the present study because LDL cholesterol was indirectly assessed based on values of total cholesterol, triglycerides, and HDL cholesterol. Non-fasting samples were also investigated for C-reactive protein (CRP), HDL cholesterol, and total cholesterol. After resting quietly in a sitting position for $5 \mathrm{~min}$, three or more blood pressure determinations (systolic and diastolic) were obtained. The average of the obtained systolic and diastolic measurements was used. Additionally, anthropometric measurements including waist circumference, triceps skinfold, subscapularis skinfold, height, and weight were also obtained. BMI was calculated from measured weight and height (weight in kilograms divided by the square of height in meters). These biologically related variables were chosen because they have previously been shown to be associated with physical activity [13-16]. Further details about the laboratory and examination procedures and quality control have been reported elsewhere [17].

\section{Measurement of Physical Activity}

The physical activity monitoring component was first added to the NHANES 2003-2004 cycle and was also included in the NHANES 2005-2006 cycle. To increase the sample size and statistical power, we combined accelerometry data from both 2-year cycles. At the MEC, participants not limited by impairments of walking or wearing an accelerometer were recruited to wear an ActiGraph 7164 accelerometer. Following their examination, participants were asked to wear the accelerometer during all waking hours, positioned on the right hip on an elasticized fabric belt, over a 7-day period. Participants were instructed to remove the accelerometer while involved in any water-based activities (e.g., showering). Accelerometry data were reduced using the SAS macro provided by the National Cancer Institute [18]. For the present study, activity counts were summarized in 1-min epoch intervals. A weighted average of 4 accelerometer-derived intensity-related count cut-points was used to classify MVPA [19]. The threshold for MVPA was 2,020 counts/min. Activity counts between 100 and 2,019 were used to classify time spent in light-intensity physical activity. Lightintensity physical activity and MVPA are reported for 1-min bout lengths. Only participants with at least 4 days of 10 or more hours per day of monitoring were included [19]. Nonwear was defined by a period of a minimum of 60 consecutive minutes of zero activity counts, with the allowance of 1-2 min of activity counts between 0 and 100 .

To examine the potential dose-response relationship between objectively measured physical activity and the study variables, physical activity was categorized into deciles (i.e., 10 groups) of light-intensity physical activity and MVPA. This allowed for the examination of physical activity levels below, near, and above current recommendations (i.e., $150 \mathrm{~min} /$ week of MVPA).

\section{Measurement of Demographic Variables}

Age (continuous), gender (male/female), smoking status (current smoker, previous smoker, and never smoked), and race-ethnicity (Mexican American, non-Hispanic whites, and non-Hispanic Blacks) were assessed from data collected using a questionnaire administered during the household interview. Trained 
Loprinzi et al:: Dose Response Association Between Physical Activity and Biological, Demographic, and Perceptions of Health Variables

household interviewers administered the questionnaire with interview data recorded using a Blaise format computer-assisted personal interview (CAPI) system. These demographic variables were chosen as covariates or to follow up on the work of Hawkins and colleagues [8]. Further details are available elsewhere [20].

\section{Measurement of Mental and Physical Health Variables}

Given the link between health perception and indices of cardiovascular disease, mental and physical health perception variables were assessed. At the MEC, a trained interviewer used the CAPI system to ask questions about the participant's perception of their mental and physical health. Participants were asked the following questions: i) 'Think about your physical health, which includes physical illness and injury, for how many days during the past 30 days was your physical health not good?'; ii) 'Now think about your mental health, which includes stress, depression, and problems with emotions, for how many days during the past 30 days was your mental health not good?'; and iii) 'During the past 30 days, for about how many days did poor physical or mental health keep you from doing usual activities, such as self-care, work, school or recreation?'. These variables were included given the link between perceptions of health and physical activity $[9,11]$.

\section{Covariates}

Covariates used to produce adjusted means included age, measured BMI, gender, race-ethnicity, smoking, SES, and history of coronary heart disease and stroke. SES was ascertained using the poverty-toincome ratio. Ranging from 0 to 5 , the poverty-to-income ratio was defined as the ratio of the family individual income to their poverty threshold. With respect to history of chronic disease, participants were asked whether they were ever told by their doctor that they had coronary heart disease or a stroke.

\section{Data Analysis}

Statistical analyses were performed using procedures from sample survey data using STATA to account for the complex NHANES survey design. To account for oversampling and non-response, all analyses included the use of appropriate sample weights, clustering, and primary sampling unit variables. New sample weights were created for the combined NHANES cycles following analytical guidelines for the continuous NHANES. Weighted mean (standard error) minutes (for each intensity) in each decile along with the weighted proportion (standard error) of participants in each decile were calculated. Adjusted means (95\% confidence intervals $(95 \% \mathrm{CIs})$ ) were calculated for the biological and perception of physical and mental health variables. Means were adjusted for age, BMI, gender, race-ethnicity, smoking, SES, and history of coronary heart disease or stroke. Those who self-reported taking medication for hypertension, cholesterol, or diabetes had their scores adjusted for. Statistical differences between the continuous variables across the deciles of physical activity were tested using an adjusted Wald test. Statistical differences between categorical variables were tested with design-based likelihood ratio chi-square tests. Multivariate logistic regression analysis was used to examine the association between each of the physical activity classifications and the odds of having metabolic syndrome. The first decile group was the reference group. Covariates for the logistic regression included age, gender, history of coronary heart disease or stroke, race-ethnicity, SES, and smoking status. Statistical significance was established as $\mathrm{p} \leq 0.01$.

\section{Results}

Table 1 provides information on the weighted percent of individuals in each physical activity classification, as well as the weighted mean light physical activity and MVPA in each classification.

\section{Primary Outcome: Metabolic Syndrome (and Related Biological Variables)}

The weighted prevalence estimate of metabolic syndrome among US adults was $38.6 \%$. For light-intensity physical activity, those in decile 9 had the lowest odds of having metabolic syndrome $(\mathrm{OR}=0.55 ; 95 \% \mathrm{CI} 0.35-0.86 ; \mathrm{p}<0.05)$ (tables 2, 3). For MVPA, participants in decile 10 (i.e., engaging in at least $71 \mathrm{~min} /$ day of MVPA), compared to those in decile 1 , were $80 \%$ less likely to have metabolic syndrome $(\mathrm{OR}=0.20,95 \%$ CI $0.10-0.40 ; \mathrm{p}<0.01$; tables 2 , 3). Deciles 7-9 were also significant for MVPA. 
Loprinzi et al.: Dose Response Association Between Physical Activity and Biological, Demographic, and Perceptions of Health Variables

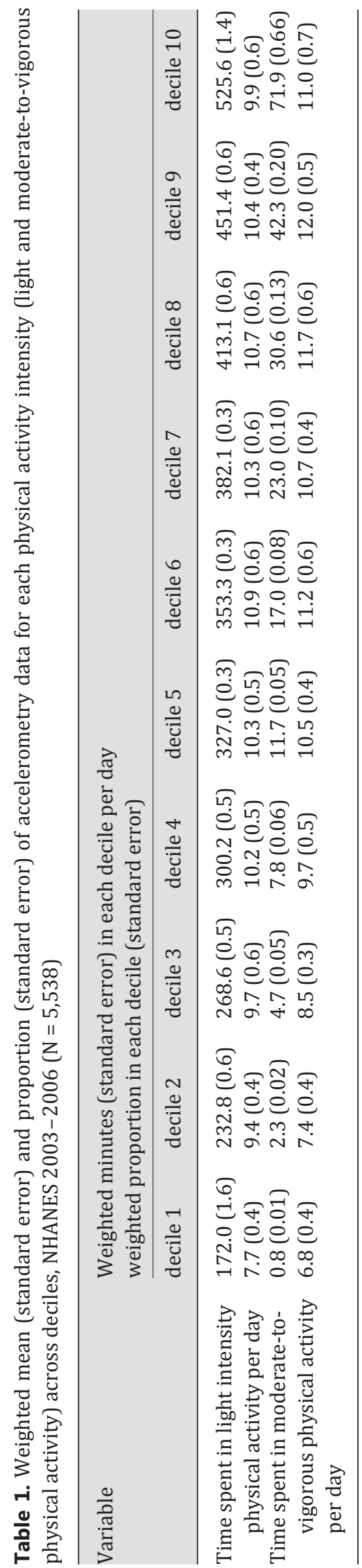




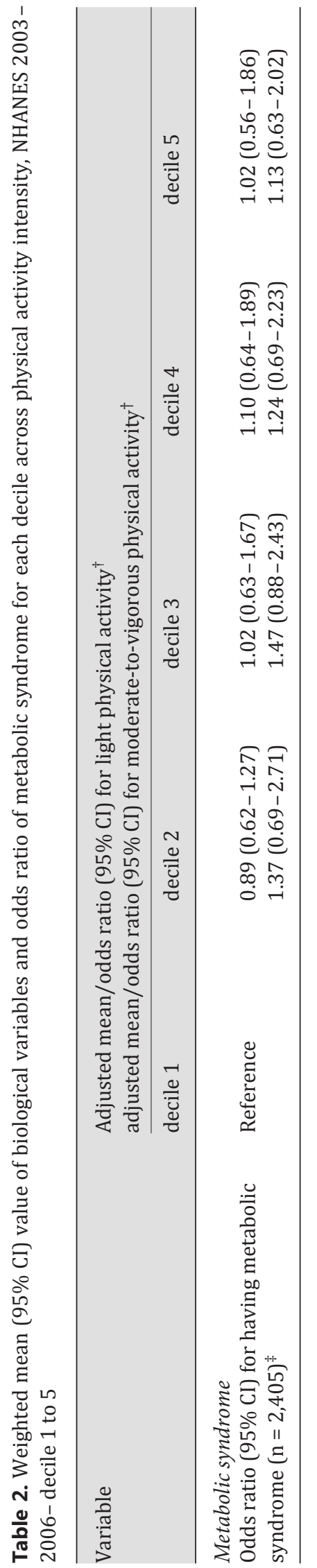

สุ

กิซึ

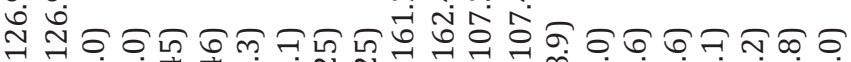
ง mं

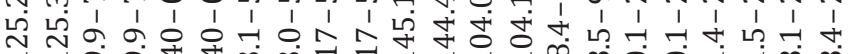

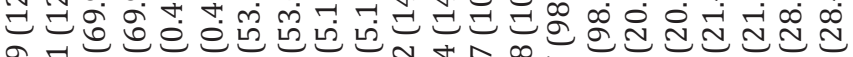

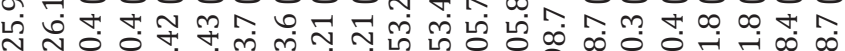

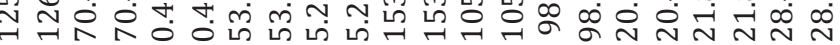

อง

กิซึ

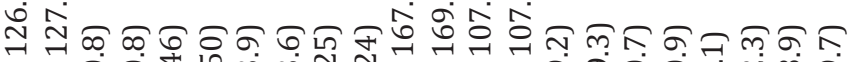

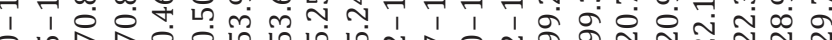
ㄴ.

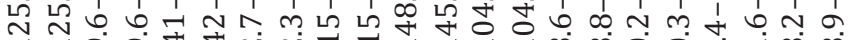

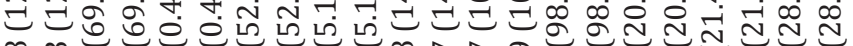

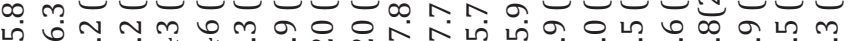

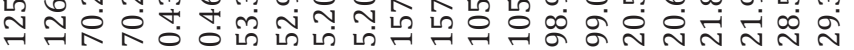

เกอ

\section{อำอ}

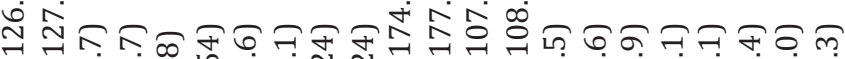
b نُ

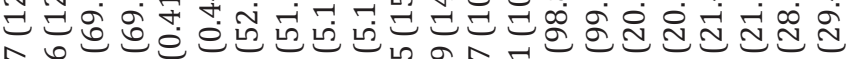

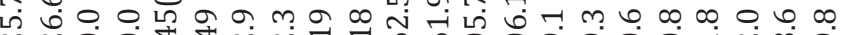
嵌 ث્才

นุำ ล๑๐็

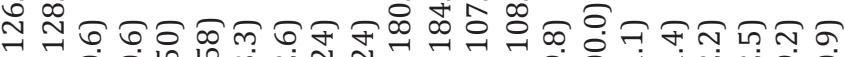

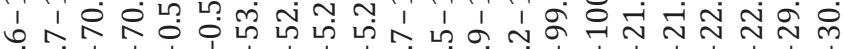
雍

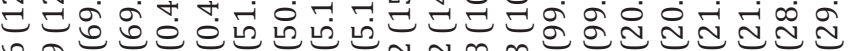

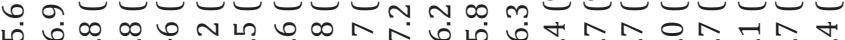
ㄱ ㄱํㅇ

กุด

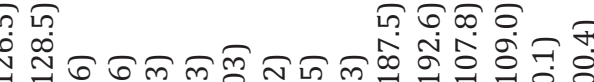
1 0 <约 H

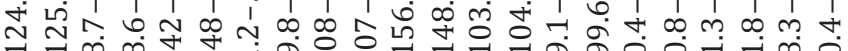

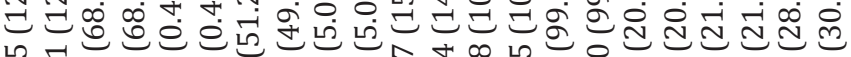

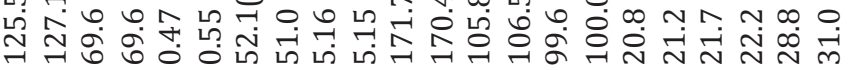

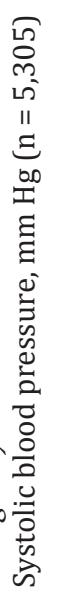

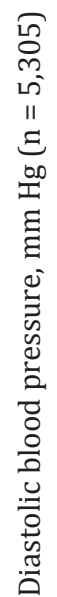
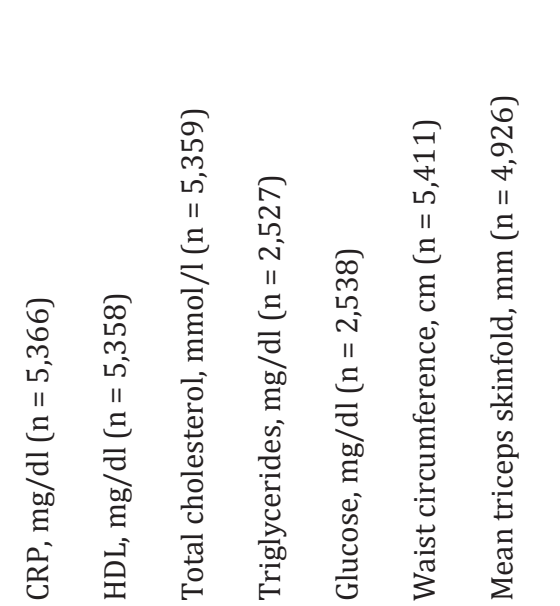

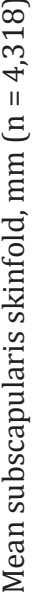

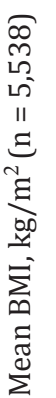

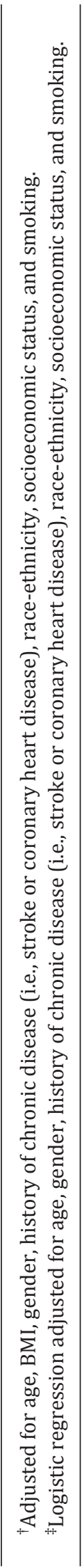




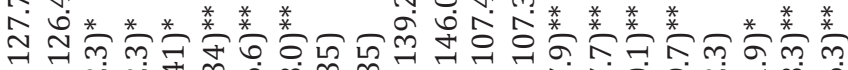
1

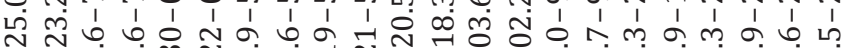

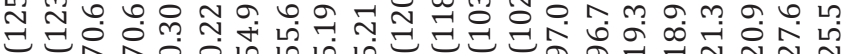

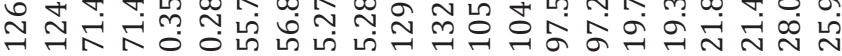

号尔

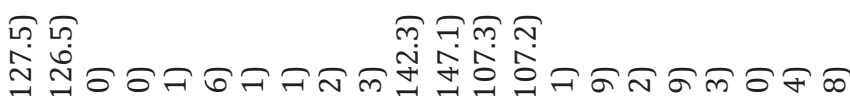

$\stackrel{*}{\infty} \underset{0}{\stackrel{*}{*}}$

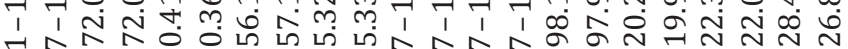
岗

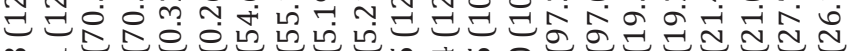
Ln

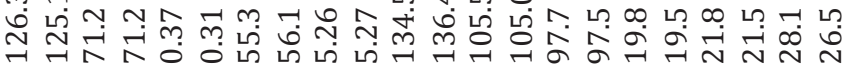

สุก กุก สกำ

ঙ $\underset{\text { F }}{\stackrel{*}{*}}$

กั่

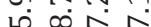

$\stackrel{m}{\rightarrow} \stackrel{0}{0}$

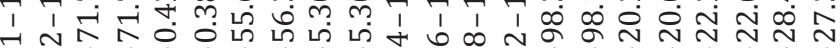

N

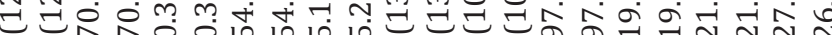

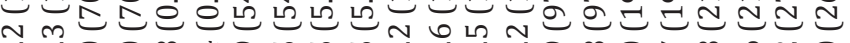

守 넉 엇

帒

*

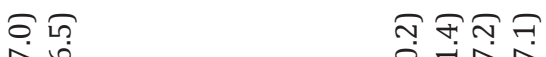

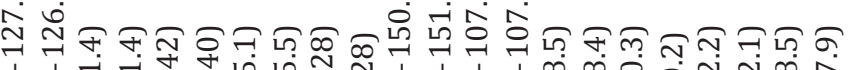

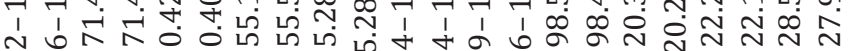

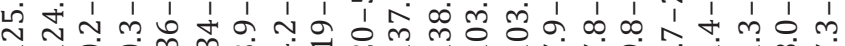

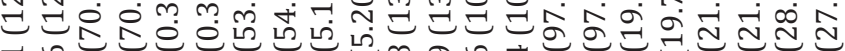

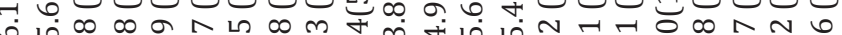

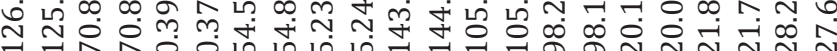

$\infty$

ฬ

20

ஸิ ข

岮通

1 1

\begin{tabular}{ll}
1 \\
1 \\
\hline
\end{tabular} न 0. ヘ

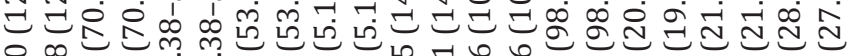

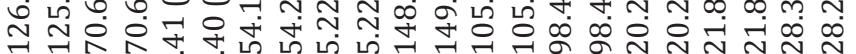

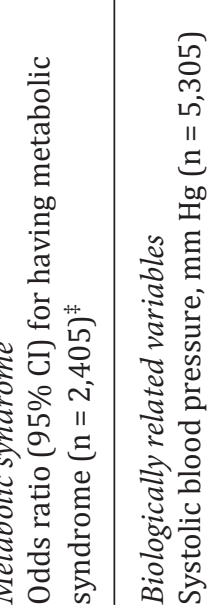

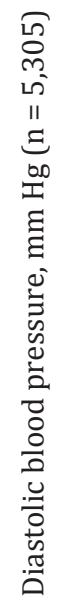
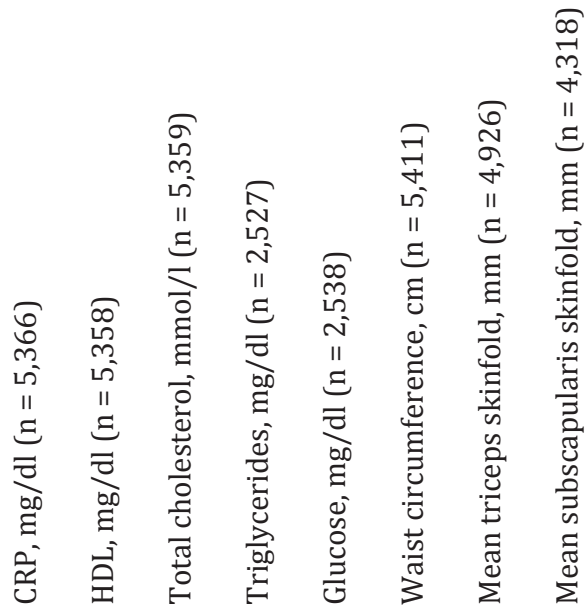

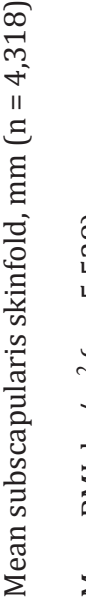

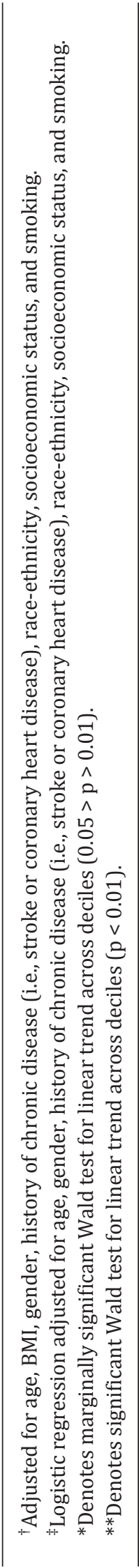


Loprinzi et al:: Dose Response Association Between Physical Activity and Biological, Demographic, and Perceptions of Health Variables

Table 4. Weighted mean/proportion (95\% CI) value of demographic variables for each decile across physical activity intensity, NHANES 2003-2006-decile 1 to $5^{\text {a }}$

\begin{tabular}{|c|c|c|c|c|c|}
\hline \multirow[t]{2}{*}{ Demographic variables } & \multicolumn{5}{|c|}{$\begin{array}{l}\text { Unadjusted mean (95\% CI) for light physical activity } \\
\text { unadjusted mean }(95 \% \mathrm{CI}) \text { for moderate-to-vigorous physical activity }\end{array}$} \\
\hline & decile 1 & decile 2 & decile 3 & decile 4 & decile 5 \\
\hline \multicolumn{6}{|l|}{ Age } \\
\hline \multirow[t]{2}{*}{ Mean age, years $(n=5,538)$} & $59.5(56.9-62.0)$ & $53.0(50.7-55.3)$ & $52.0(49.5-54.5)$ & $48.9(47.0-50.7)$ & $48.6(46.9-50.3)$ \\
\hline & $71.2(69.8-72.6)$ & $62.3(60.9-63.7)$ & $54.0(51.9-56.1)$ & $51.0(49.6-52.5)$ & $47.0(45.6-48.5)$ \\
\hline \multicolumn{6}{|l|}{ Race-ethnicity $(\mathrm{n}=5,538)^{* *}$} \\
\hline \multirow[t]{2}{*}{ \% Mexican Americans } & $3.2(2.4-4.0)$ & $4.8(3.7-6.0)$ & $6.8(5.0-8.6)$ & $7.4(5.4-9.3)$ & $7.8(5.7-9.9)$ \\
\hline & $2.3(1.5-3.2)$ & $5.2(3.5-6.9)$ & $7.3(5.7-8.8)$ & $8.5(6.3-10.7)$ & $10.9(8.4-13.4)$ \\
\hline \multirow[t]{2}{*}{ \% other Hispanic } & $2.8(0.5-5.1)$ & $4.9(0.4-9.4)$ & $4.6(0.2-9.0)$ & $14.6(8.4-20.7)$ & $8.5(3.2-13.7)$ \\
\hline & $2.1(-0.06-4.4)$ & $0.8(-0.2-1.9)$ & $5.7(0.6-10.8)$ & $9.3(4.3-14.2)$ & $11.7(7.3-16.2)$ \\
\hline \multirow[t]{2}{*}{ \% Whites } & $8.4(7.3-9.6)$ & $10.1(8.8-11.4)$ & $10.3(8.6-12.0)$ & $10.5(9.2-11.7)$ & $10.6(9.2-12.0)$ \\
\hline & $7.9(6.8-9.0)$ & $7.7(6.7-8.8)$ & $8.2(7.3-9.8)$ & $9.6(8.1-11.1)$ & $9.9(8.6-11.2)$ \\
\hline \multirow[t]{2}{*}{$\%$ Blacks } & $6.4(5.0-7.9)$ & $8.7(7.3-10.0)$ & $8.5(6.5-10.4)$ & $9.2(7.3-11.1)$ & $11.0(9.0-13.0)$ \\
\hline & $5.6(3.6-7.6)$ & $9.6(7.6-11.6)$ & $10.0(8.0-12.1)$ & $11.0(9.0-12.9)$ & $12.6(10.1-15.0)$ \\
\hline \multirow[t]{2}{*}{$\%$ other race } & $10.0(3.3-16.6)$ & $9.9(5.6-14.3)$ & $10.9(5.5-16.2)$ & $9.1(4.6-13.5)$ & $9.0(5.2-12.7)$ \\
\hline & $3.6(0.6-6.7)$ & $5.8(2.3-9.4)$ & $13.2(7.9-18.4)$ & $11.0(6.4-15.7)$ & $13.1(8.0-18.1)$ \\
\hline \multicolumn{6}{|l|}{ Gender $(n=5,538)^{* *}$} \\
\hline \multirow[t]{2}{*}{$\%$ males } & $8.4(7.0-9.8)$ & $10.0(8.6-11.5)$ & $10.3(8.7-11.9)$ & $9.3(8.0-10.6)$ & $9.1(7.9-10.3)$ \\
\hline & $4.6(3.6-5.5)$ & $5.0(4.2-5.8)$ & $5.9(4.9-6.8)$ & $7.0(5.9-8.0)$ & $8.7(7.2-10.2)$ \\
\hline \multirow[t]{2}{*}{$\%$ females } & $7.1(5.9-8.2)$ & $8.8(7.6-10.0)$ & $9.1(7.4-10.9)$ & $11.0(9.3-12.7)$ & $11.4(9.4-13.5)$ \\
\hline & $9.0(7.7-10.4)$ & $9.8(8.4-11.1)$ & $11.0(9.9-12.2)$ & $12.310 .8-13.9)$ & $12.2(10.6-13.8)$ \\
\hline
\end{tabular}

Tables 2 and 3 also show the weighted and adjusted means for the biological variables. For light-intensity physical activity, an inverse association and a significant test for linear trend was found for CRP, triglycerides, waist circumference, triceps skinfold, and BMI. Notably, for diastolic blood pressure and HDL cholesterol, a positive association was found. For MVPA, an inverse association and a significant test for linear trend was found for CRP, triglycerides, waist circumference, triceps and subscapularis skinfold, and BMI. A positive association was found for HDL cholesterol and diastolic blood pressure.

\section{Secondary Outcomes: Demographics and Mental/Physical Health Perceptions}

Tables 4 and 5 present the weighted unadjusted mean or proportion for the demographic variables. As expected, there was an inverse association between age and objectively measured physical activity, with younger participants engaging in more MVPA per week than older participants $(\mathrm{p} \leq 0.01)$. With respect to race-ethnicity, Mexican Americans and Other Hispanics, compared to non-Hispanic Whites, non-Hispanic Blacks, and other races, spent more time in MVPA $(\mathrm{p}<0.0001)$. A similar trend was found for light-intensity physical activity.

Males were more physically active than females ( $\mathrm{p} \leq 0.01$ ), with $17.6 \%$ (95\% CI 15.0$20.1 \%$ ) engaged in at least $71 \mathrm{~min} /$ day of MVPA compared to $4.6 \%$ (95\% CI 3.5-5.7\%). A similar trend was found for light-intensity physical activity.

Table 6 presents the weighted and adjusted means for the mental/physical health perceptional variables. With respect to the participants' perception of their mental and physical health status, participants who were more active had fewer number of days where they perceived their physical ( $p \leq 0.01$ ) or mental ( $p \leq 0.01$ ) health to not be good, and they also had fewer number of inactive days attributable to their physical or mental health $(p<0.01)$. 
Loprinzi et al.: Dose Response Association Between Physical Activity and Biological, Demographic, and Perceptions of Health Variables

Table 5. Weighted mean/proportion (95\% CI) value of demographic variables for each decile across physical activity intensity, NHANES 2003-2006 - decile 6 to $10^{\mathrm{a}}$

\begin{tabular}{|c|c|c|c|c|c|}
\hline \multirow[t]{2}{*}{ Demographic variables } & \multicolumn{5}{|c|}{$\begin{array}{l}\text { Unadjusted mean }(95 \% \mathrm{CI}) \text { for light physical activity } \\
\text { unadjusted mean }(95 \% \mathrm{CI}) \text { for moderate-to-vigorous physical activity }\end{array}$} \\
\hline & decile 6 & decile 7 & decile 8 & decile 9 & decile 10 \\
\hline \multicolumn{6}{|l|}{ Age } \\
\hline Mean age, years & $47.1(45.4-48.8)$ & $46.1(44.4-47.8)$ & $44.3(42.7-45.9)$ & $44.6(43.3-45.8)$ & $42.5(41.0-44.0)^{* *}$ \\
\hline$(\mathrm{n}=5,538)$ & $45.5(44.0-46.9)$ & $44.5(43.4-45.6)$ & $42.2(40.8-43.6)$ & $41.7(40.1-43.2)$ & $39.4(38.1-40.7)^{* *}$ \\
\hline \multicolumn{6}{|c|}{ Race-ethnicity $(\mathrm{n}=5,538)^{* *}$} \\
\hline \multirow[t]{2}{*}{ \% Mexican Americans } & $8.4(6.8-10.0)$ & $11.9(10.5-13.4)$ & $14.3(11.6-16.9)$ & $14.9(12.7-17.1)$ & $20.0(16.3-23.7)$ \\
\hline & $10.0(8.0-12.0)$ & $11.7(9.1-14.3)$ & $10.6(8.2-13.0)$ & $14.7(12.2-17.2)$ & $18.2(16.0-20.5)$ \\
\hline \multirow[t]{2}{*}{$\%$ other Hispanic } & $8.0(2.3-13.6)$ & $10.4(3.3-17.4)$ & $13.9(6.0-21.8)$ & $16.2(9.6-22.8)$ & $15.8(10.7-20.9)$ \\
\hline & $8.6(3.2-14.0)$ & $16.6(8.4-24.8)$ & $10.9(5.3-16.4)$ & $14.6(8.4-20.9)$ & $19.2(12.0-26.4)$ \\
\hline \multirow[t]{2}{*}{$\%$ Whites } & $11.3(10.0-12.7)$ & $10.1(8.6-11.5)$ & $10.1(8.5-11.7)$ & $9.5(8.4-10.6)$ & $8.5(6.9-10.2)$ \\
\hline & $11.1(9.8-12.4)$ & $10.3(9.3-11.3)$ & $12.5(10.8-14.2)$ & $12.0(10.6-13.4)$ & $10.3(8.4-12.1)$ \\
\hline \multirow[t]{2}{*}{$\%$ Blacks } & $10.0(8.6-11.4)$ & $11.7(9.4-14.0)$ & $10.2(8.0-12.3)$ & $12.4(10.2-14.5)$ & $11.5(9.2-13.9)$ \\
\hline & $10.2(8.4-12.0)$ & $11.7(9.9-13.5)$ & $9.4(7.2-11.6)$ & $9.7(7.5-12.0)$ & $9.7(7.6-11.8)$ \\
\hline \multirow[t]{2}{*}{$\%$ other race } & $11.2(5.2-17.2)$ & $9.0(4.3-13.8)$ & $13.0(8.0-18.0)$ & $9.5(5.1-13.9)$ & $8.0(4.4-11.5)$ \\
\hline & $17.9(10.5-25.4)$ & $9.9(5.3-14.6)$ & $7.0(3.6-10.5)$ & $10.5(6.1-14.8)$ & $7.4(3.4-11.4)$ \\
\hline \multicolumn{6}{|l|}{ Gender $(n=5,538)^{* *}$} \\
\hline \multirow[t]{2}{*}{$\%$ males } & $9.8(8.3-11.2)$ & $9.3(7.6-11.0)$ & $11.1(9.1-13.0)$ & $10.3(8.8-11.8)$ & $11.9(10.5-13.4)$ \\
\hline & $10.3(8.8-11.8)$ & $11.2(10.0-12.4)$ & $13.7(12.3-15.1)$ & $15.6(13.5-17.7)$ & $17.6(15.0-20.1)$ \\
\hline \multirow[t]{2}{*}{$\%$ females } & $11.9(10.3-13.6)$ & $11.39 .6-13.0)$ & $10.3(8.8-11.8)$ & $10.5(9.1-12.0)$ & $7.9(6.5-9.4)$ \\
\hline & $12.1(10.5-13.6)$ & $10.2(8.8-11.6)$ & $9.8(8.0-11.6)$ & $8.5(7.0-9.9)$ & $4.6(3.5-5.7)$ \\
\hline
\end{tabular}

\section{Discussion}

The present study was unique in that we used all available objectively measured physical activity data from NHANES and examined the potential dose-response relationship between physical activity and key biological, demographic, and health perception variables among a nationally representative sample of US adults across ten physical activity doses. Our main finding was that health benefits associated with physical activity generally increased with each increasing level of physical activity, suggesting that increasing an individual's activity behavior may elicit improvements in a variety of health parameters. Importantly, engaging in higher levels of either light- or higher-intensity physical activity may elicit health benefits. Although more favorable levels of health outcomes were associated with increasing levels of light-intensity physical activity and MVPA , participants who participated in at least 71 min/day of MVPA (over three times the current weekly physical activity recommendation) were the least likely to have metabolic syndrome. In support of the positive benefits of higher levels of light-intensity physical activity and MVPA, participants who engaged in at least 71 min/day of MVPA had the lowest CRP concentrations, triglyceride levels, waist circumference, triceps and subscapularis measurements, and BMI as well as the highest levels of HDL cholesterol. Similar results were also found for those engaging in at least $525 \mathrm{~min} /$ day of light- 


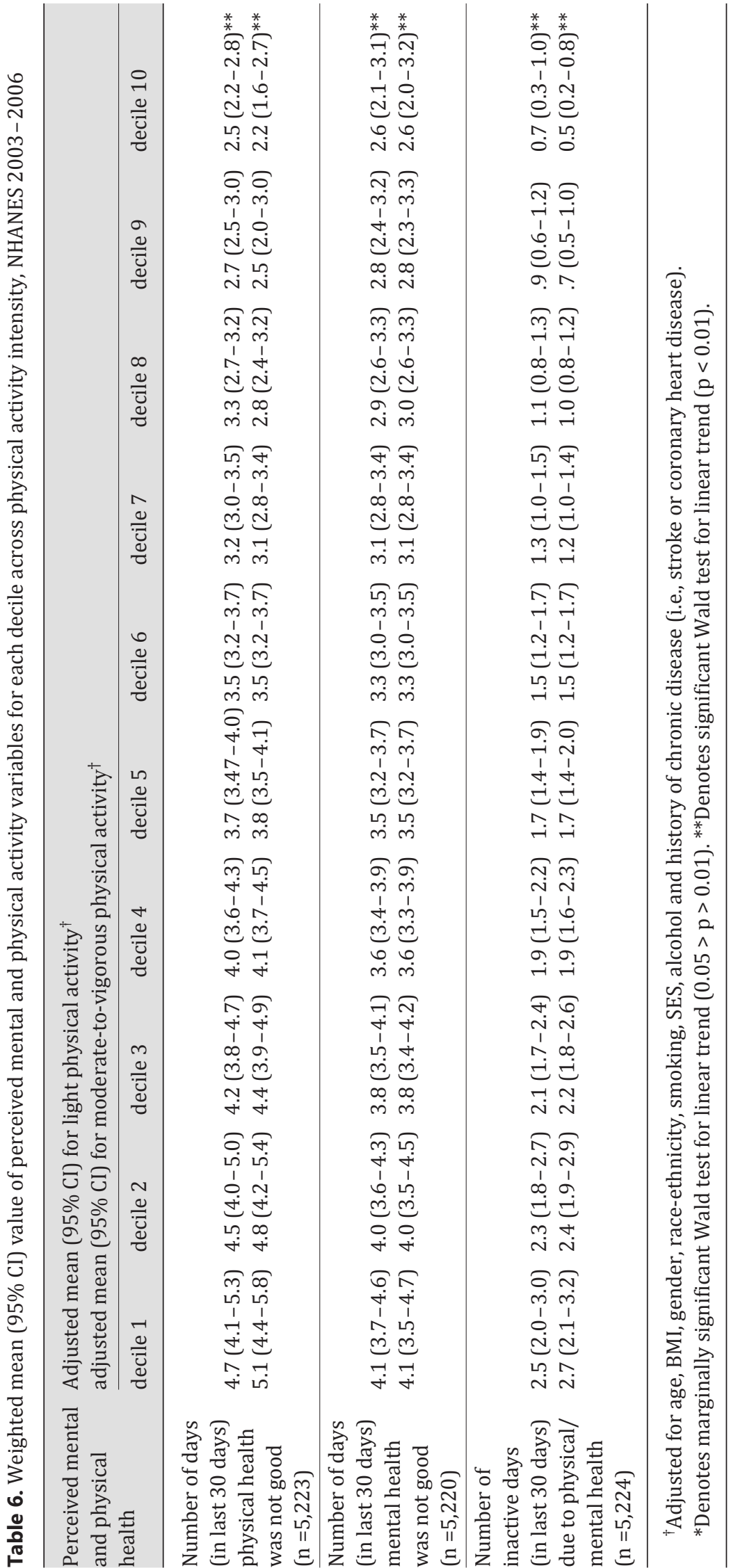


Loprinzi et al.: Dose Response Association Between Physical Activity and Biological, Demographic, and Perceptions of Health Variables

intensity physical activity. Although difficult to explain, there was a positive association between physical activity and diastolic blood pressure; however, the difference between the lowest and highest decile was $2 \mathrm{~mm}$ Hg. Future experimental and prospective studies are needed to determine whether these high doses of light-intensity physical activity and MVPA per day, or perhaps even a higher dose of physical activity, cause additional health benefits, or alternatively, whether even higher levels of physical activity result in a diminishing effect, possibly due to an increased likelihood of injury.

Findings from Metzger et al. [7] showed that generally higher levels of physical activity were associated with lower odds of being classified with any of the risk factors, with perhaps the greatest benefit occurring in those who engaged in roughly twice the current physical activity recommendations (i.e., approximately $300 \mathrm{~min} /$ week of MVPA). Our findings extend these findings by also showing an association between light-intensity physical activity and cardiovascular disease risk factors as well as suggest that even higher levels of physical activity of MVPA (i.e., $71 \mathrm{~min} /$ day of MVPA or nearly $500 \mathrm{~min} /$ week of MVPA) may result in even more favorable health outcomes. Results from a recent meta-analysis by Sattelmair et al. [21] are in accordance with the present study. Sattelmair and colleagues [21] reviewed 33 epidemiological prospective cohort studies investigating the association between physical activity and coronary heart disease. Across the studies, individuals who engaged in the equivalent of $150 \mathrm{~min} /$ week of moderate-intensity leisure time physical activity had a 14\% lower coronary heart disease risk. Participants engaging in 300 min of moderate-intensity leisure time physical activity per week had a $20 \%$ lower risk. Higher levels of physical activity also resulted in a modestly lower risk. With respect to findings from blood pressure-related randomized controlled trials, Cornelissen and Fagard [22] performed a comprehensive metaanalysis examining the effect of endurance training on cardiovascular disease risk. In support of the present study, their findings showed that, among the 72 trials, endurance training (median duration 16 weeks; median frequency 3 days/week; median duration of training session 40 min; median training intensity $65 \%$ of heart rate reserve) was effective in reducing body weight and waist circumference and increasing HDL cholesterol. Leon and Sanchez [23] conducted a meta-analysis examining the influence of aerobic-based intervention trials or randomized controlled trials on blood lipids. Among the 51 studies that employed an exercise regimen similar to the American College of Sports Medicine guidelines (i.e., moderate to vigorous intensity, 3-5 days/week for 30 min or more per session), exercise training was consistently positively associated with HDL cholesterol, but inconsistently associated with reductions in total cholesterol, LDL cholesterol, and triglycerides. Additional experimental studies are needed to determine what dose of physical activity results in the most optimal health outcome and whether this is dependent on the individual, as individuals seem to differentially adapt to exercise $[24,25]$.

We also examined doses of objectively measured physical activity by age, gender, and race-ethnicity. Our findings with respect to these objectives revealed: i) there was a clear dose-response relationship between age and physical activity, showing that older individuals engaged in less physical activity than younger individuals; ii) at nearly all of the higher doses of physical activity, a greater percent of Mexican American and other Hispanic adults engaged in more physical activity compared to non-Hispanic Whites, non-Hispanic Blacks and other races; and iii) at all of the higher doses of physical activity, males were more active than females. These findings are in support of those by Hawkins et al. [8] who showed that male Hispanics (303.9) had higher average daily activity counts (not to be interpreted as MVPA) than male Whites (258.0) and male Blacks (266.4). Similar results were observed for females in that female Hispanics (217.9) had higher average daily activity counts than female Whites (206.2) and female Blacks (192.6). The findings by Hawkins et al. [8], along with those of the present study, show that males are more active than females, that Hispanics are more active 
Loprinzi et al:: Dose Response Association Between Physical Activity and Biological, Demographic, and Perceptions of Health Variables

than Whites and Blacks, and that these differences exist at nearly all of the higher doses of physical activity.

Lastly, we examined the dose-response relationship between objectively measured physical activity and perceptions of physical and mental health status. We found a graded relationship between physical activity and self-rated health in that those with higher levels of physical activity generally had fewer days where their perceived physical or mental health was not good, had fewer inactive days, and perceived themselves to have excellent health compared to those in the lower doses of physical activity. These findings are similar to those of Tsai and colleagues [10] who showed that, among 430,912 adults 18 years and older from the Behavioral Risk Factor Surveillance System, individuals with type 1 diabetes, type 2 diabetes and without diabetes who self-reported being active had an increased likelihood of $81 \%, 32 \%$ and $18 \%$, respectively, of reporting optimal self-rated health, compared with adults who self-reported being inactive. These results are consistent with those of Bertheussen et al. [11] who showed that, among 4,500 participants (mean age $53 \pm 15$ years), any level of self-reported physical activity was associated with better physical and mental health in both genders when compared with no exercise. These studies support the need to counsel adults with lower self-perceptions of health regarding how to integrate physical activity into their lifestyle [26].

A limitation of this study is its cross-sectional study design, which does not allow for the evaluation of a cause-and-effect relationship. As a result, we are not able to determine whether individuals who are more active have more favorable health outcomes or, conversely, whether healthier individuals are more able and likely to be physically active. Major strengths of the study include using a nationally representative sample of US adults, the objective measurement of light-intensity physical activity and MVPA, combining both NHANES cycles to increase the sample size, and using objective measures of biological outcomes.

In conclusion, the major finding of this study is that individuals who engaged in nearly $500 \mathrm{~min} /$ week of MVPA had more favorable health outcomes, including lower odds of developing metabolic syndrome. Importantly, engaging in high levels of light-intensity physical activity was also associated with more favorable cardiovascular disease risk factors. Additionally, males and Hispanics are more active at the higher doses of physical activity than their counterparts, and that generally higher levels of physical activity are associated with better self-perceptions of physical, mental, and overall health.

\section{Disclosure Statement}

The authors declare no conflict of interest.

\section{References}

1 Heron MP, Hoyert DL, Murphy A, Xu JQ, Kochanek KD, Tejada-Vera B: Deaths: Final Data for 2006; in National Vital Statistics Reports, Hyattsville, National Center for Health Statistics, 2009.

-2 Wilson PW, D'Agostino RB, Parise H, Sullivan L, Meigs JB: Metabolic syndrome as a precursor of cardiovascular disease and type 2 diabetes mellitus. Circulation 2005;112:3066-3072.

- 3 Pitsavos C, Panagiotakos D, Weinem M, Stefanadis C: Diet, exercise and the metabolic syndrome. Rev Diabet Stud 2006;3:118-126.

4 Carnethon MR, Loria CM, Hill JO, Sidney S, Savage PJ, Liu K: Risk factors for the metabolic syndrome: the Coronary Artery Risk Development in Young Adults (CARDIA) study, 1985-2001. Diabetes Care 2004;27: 2707-2715.

5 Churilla JR, Zoeller RF: Physical activity and the metabolic syndrome: a review of evidence. Am J Lifestyle Med 2008;2:118-125. 
Loprinzi et al.: Dose Response Association Between Physical Activity and Biological, Demographic, and Perceptions of Health Variables

-6 Loprinzi PD, Cardinal BJ, Crespo C, Brodowicz GR, Andersen R, Smit E: Objectively-measured physical activity and C-reactive protein: National Health and Nutrition Examination Survey 2003-2004. Scand J Med Sci Sports 2013;23:164-170.

7 Metzger JS, Catellier D, Evenson KR, Treuth M, Rosamond WD, Siega-Riz AM: Associations between patterns of objectively measured physical activity and risk factors for metabolic syndrome. Am J Health Promot 2010; 24:161-169.

-8 Hawkins MS, Storti KL, Richardson CR, King WC, Strath SJ, Holleman RG, Kriska AM: Objectively measured physical activity of USA adults by sex, age, and racial/ethnic groups: a cross-sectional study. Int J Behav Nutr Phys Act 2009;6:31.

-9 Price AE, Corwin SJ, Friedman DB, Laditka SB, Colabianchi N, Montgomery KM: Older adults' perceptions of physical activity and cognitive health: implications for health communication. Health Educ Behav 2011;38: $15-24$.

10 Tsai J, Ford ES, Li C, Zhao G, Balluz LS: Physical activity and optimal self-rated health of adults with and without diabetes. BMC Public Health 2010;10:365.

11 Bertheussen GF, Romundstad PR, Landmark T, Kaasa S, Dale O, Helbostad JL: Associations between physical activity and physical and mental health - a HUNT 3 Study. Med Sci Sports Exerc 2011;43:1220-1228.

$\checkmark 12$ Grundy SM, Cleeman JI, Daniels SR, Donato KA, Eckel RH, Franklin BA, Gordon DJ, Krauss RM, Savage PJ, Smith SC Jr, Spertus JA, Fernando C: Diagnosis and management of the metabolic syndrome: an American Heart Association/National Heart, Lung, and Blood Institute scientific statement: executive summary. Crit Pathw Cardiol 2005; 4:198-203.

13 Lokey EA Tran ZV: Effects of exercise training on serum lipid and lipoprotein concentrations in women: a meta-analysis. Int J Sports Med 1989;10:424-429.

14 Plaisance EP Grandjean PW: Physical activity and high-sensitivity C-reactive protein. Sports Med 2006;36: 443-458.

15 Kronenberg F, Pereira MA, Schmitz MK, Arnett DK, Evenson KR, Crapo RO, Jensen RL, Burke GL, Sholinsky P, Ellison RC, Hunt SC: Influence of leisure time physical activity and television watching on atherosclerosis risk factors in the NHLBI Family Heart Study. Atherosclerosis 2000;153:433-443.

-16 Vianna MV, Ali Cader S, Gomes AL, Guimaraes AC, Seixas-da-Silva IA, do Rego AR, Adao KD, Dantas EH: Aerobic conditioning, blood pressure (BP) and body mass index (BMI) of older participants of the Brazilian Family Health Program (FHP) after 16 weeks of guided physical activity. Arch Gerontol Geriatr 2012;54:210-213.

17 Centers for Disease Control and Prevention: National Health and Nutrition Examination Survey (NHANES 2003-2004): Laboratory Manual. Bethesda, National Center for Health Statistics. www.cdc.gov/nchs/data/ nhanes/nhanes_03_04/l11_c.pdf.

-18 Centers for Disease Control and Prevention: National Health and Nutrition Examination Survey (NHANES 2003-2004): National Cancer Institute. www.cdc.gov/nchs/nhanes/nhanes2003-2004/exam03_04.htm.

19 Troiano RP, Berrigan D, Dodd KW, Masse LC, Tilert T, McDowell M: Physical activity in the United States measured by accelerometer. Med Sci Sports Exerc 2008;40:181-188.

20 Centers for Disease Control and Prevention, National Center for Health Statistics: National Health and Nutrition Examination Protocol and Questionnaire. Hyattsville, US Department of Health and Human Services, Centers for Disease Control and Prevention, 2010. www.cdc.gov/nchs/nhanes/nhanes2003-2004/nhanes03_04.htm.

-21 Sattelmair J, Pertman J, Ding EL, Kohl HW 3rd, Haskell W, Lee IM: Dose response between physical activity and risk of coronary heart disease: a meta-analysis. Circulation 2011;124:789-795.

-22 Cornelissen VA Fagard RH: Effects of endurance training on blood pressure, blood pressure-regulating mechanisms, and cardiovascular risk factors. Hypertension 2005;46:667-675.

$\checkmark 23$ Leon AS Sanchez OA: Response of blood lipids to exercise training alone or combined with dietary intervention. Med Sci Sports Exerc 2001;33:S502-515.

-24 Skinner JS, Jaskolski A, Jaskolska A, Krasnoff J, Gagnon J, Leon AS, Rao DC, Wilmore JH, Bouchard C: Age, sex, race, initial fitness, and response to training: the HERITAGE Family Study. J Appl Physiol 2001;90:1770-1776.

25 Torok DJ, Duey WJ, Bassett DR Jr, Howley ET, Mancuso P: Cardiovascular responses to exercise in sprinters and distance runners. Med Sci Sports Exerc 1995;27:1050-1056.

26 Li KK, Cardinal BJ, Vuchinich S: Health worry, physical activity participation, and walking difficulty among older adults: a mediation analysis. Res Q Exerc Sport 2009;80:12-21. 